\title{
Avaliação da modificação de um sistema composto por tanque séptico e filtro anaeróbio por um modelo de aeração compartimentada.
}

\author{
Evaluation of the modification of a system consisting of septic tank and \\ anaerobic filter for a model of compartmentalized aeration
}

\section{Josué Tadeu Leite França* | Ronaldo Stefanutti | Bruno Coraucci Filho Luccas Erickson Marinho | Gentil Tereziano Rodrigues}

Data de entrada: 23/10/2013 | Data de aprovação: 23/04/2014

DOI: http://dx.doi.org/10.4322/dae.2014.127

Resumo

Este trabalho teve como objetivo avaliar a modificação de um sistema de tratamento composto por dois tanques séptico com operação em paralelo por um modelo de aeração compartimentada. Tal proposta visa atender à obtenção da Licença de Operação (L.O) e ao padrão de lançamento. Após as adequações, foi feita a comparação dos resultados obtidos com resultados da situação inicial. A remoção de carga orgânica passou de 59\% para 95\%, com a DBO efluente em torno de $30 \mathrm{mg} / \mathrm{L}^{-1}$. A média com relação ao residual de oxigênio dissolvido no efluente foi de $1,90 \pm 1,2 \mathrm{mg} / \mathrm{L}^{-1}$, a densidade de potência 30 $\mathrm{W} / \mathrm{m}^{3}$, o consumo de energia foi de $37 \mathrm{kWh} \cdot \mathrm{hab}^{-1}$ e o custo da modificação per capta foi de R $\$ 140,00$.

Palavras-chave: Tanque séptico, Filtro anaeróbio, Modificação e Aeração.

\section{Abstract}

This work aimed to evaluate the modification of a treatment system comprising two septic tanks and two anaerobic filters whit parallel operation for a model of compartmentalized aeration. This proposal aims to meet to obtain an Operating License (LO) and the discharge parameters. After the adjustments, it was compared the results obtained and the results of the initial situation. The removal of organic matter increased from 59\% to 95\%, with an average BOD of the effluent around $30 \mathrm{mg} / \mathrm{L}^{-1}$. The average of residual dissolved oxygen in the effluent was $1,90 \pm 1.1 \mathrm{mg} / \mathrm{L}^{-1}$, the power density of $30 \mathrm{~W} \cdot \mathrm{m}^{3}$, the energy consumption was $37 \mathrm{kWh} \mathrm{inh}^{-1}$ and the cost of modification per capita was US\$ 63,00.

Key-words: Septic tank, Snaerobic filter, Modification and Aeration. 


\section{Introdução}

O emprego do processo biológico anaeróbio por meio de tanque séptico seguido de filtro anaeróbio apresenta várias vantagens quando comparado ao processo biológico aeróbio, entre os quais, menor consumo de energia, menor produção de lodo e menor área para implantação. Apresentam, no entanto desvantagens como: baixa eficiência de remoção com relação à carga orgânica ( $<70 \%)$; pouca eficiência na remoção de nutrientes ( $\mathrm{N}$ e $\mathrm{P}$ ); eventual desequilíbrio no processo exalam fortes odores que causam transtornos junto a população, cor no efluente, além de obstrução periódica do material filtrante; produz metano que se não queimado para aproveitamento energético e liberado para o ambiente é 21 vezes mais prejudicial que o $\mathrm{CO}_{2}$ em se tratando do efeito estufa (IPCC, 2001).

"Neste contexto, o presente trabalho tem como objetivo desenvolver uma alternativa para adequação de sistema constituído por tanque séptico e filtro anaeróbio, tendo em vista tratar-se de sistema amplamente utilizado para pequenas comunidades no Brasil, que venha atender à legislação vigente e às seguintes premissas: facilidade operacional, baixo custo de implantação e manutenção, menor agressão ao ambiente e atendimento às demandas de sustentabilidade.

Os tratamentos biológicos aeróbios mais utilizados são os sistemas de lodos ativados, valo de oxidação, lagoas de estabilização e suas variantes aeradas. Esses sistemas apresentam vantagens, como alta eficiência na remoção de cargas orgânica e de nutrientes, não liberam metano para o ambiente, entretanto, como desvantagens, apresentam consumo de energia, custo elevado de operação e manutenção e produzem mais lodo seco por $\mathrm{Kg}$ de DBO removida, se comparado com os processos anaeróbios.

\section{Objetivo}

Este estudo tem como objetivo promover a modificação de um sistema anaeróbio de tratamento de esgoto para um sistema aeróbio.

\section{Materiais e métodos}

A avaliação da modificação do processo de tratamento foi realizada em três etapas distintas, onde variou-se a densidade de potência aplicada nos tanques de aeração.

\subsection{Local do experimento e histórico}

A estação de tratamento de esgoto, base da condução do estudo, localiza-se no Distrito de Bom Retiro, município de Angatuba, região sudoeste do Estado de São Paulo. De acordo com os dados da CEPAGRI no ano de 2012 a temperatura média para o município foi de $20,9^{\circ} \mathrm{C}$ e o índice de pluviosidade médio foi de $1.282,2 \mathrm{~mm}$ para aquele ano.

O sistema de tratamento, era constituído por dois conjuntos paralelos de tanques séptico seguido de filtro anaeróbio. Foi projetado para atender 300 ligações, sendo que iniciou operação em 1994 com 366 ligações. Na Tabela 01 estão apresentadas as dimensões das unidades projetadas.

Nessas condições, aliada à limitação inerente ao processo, mesmo sendo operado adequadamente, o sistema apresentava uma baixa eficiência na remoção de carga orgânica.

Em 2010, com 556 ligações de esgoto, além da baixa eficiência na remoção de carga orgânica, apresentava fuga excessiva de lodo para o corpo receptor, comprometendo ainda mais a qualidade do efluente.

Após estudos técnicos preliminares, levando em conta o volume para aeração e para sedimentação, o tempo de detenção hidráulico recomendado, a necessidade de oxigênio e a carga volumétrica aplicada nos tanques facultativo e de polimento, foi elaborada a concepção de um sistema aeróbio, utilizando todas as estruturas existentes na estação de tratamento de esgoto. A Figura 1 apresenta o croqui do modelo inicial e do modelo aeróbio proposto.

\begin{tabular}{l|c|c|c|c}
\multicolumn{1}{c|}{ Unidades } & Comprimento & Largura $(\mathrm{m})$ & Profundidade Útil $(\mathrm{m})$ & Volume $\left(\mathrm{m}^{3}\right)$ por unidade \\
\hline Tanque séptico I e II & 9,90 & 3,50 & 3,60 & 98,58 \\
\hline Filtro anaeróbio 1 e 2 & 13,85 & 5,80 & 2,05 & 164,67
\end{tabular}

Fonte: Sabesp, 2010

Tabela 1 - Medidas das unidades antes da adequação da ETE Bom Retiro 


\section{SISTEMA IMPLANTADO NO ANO DE 1994}

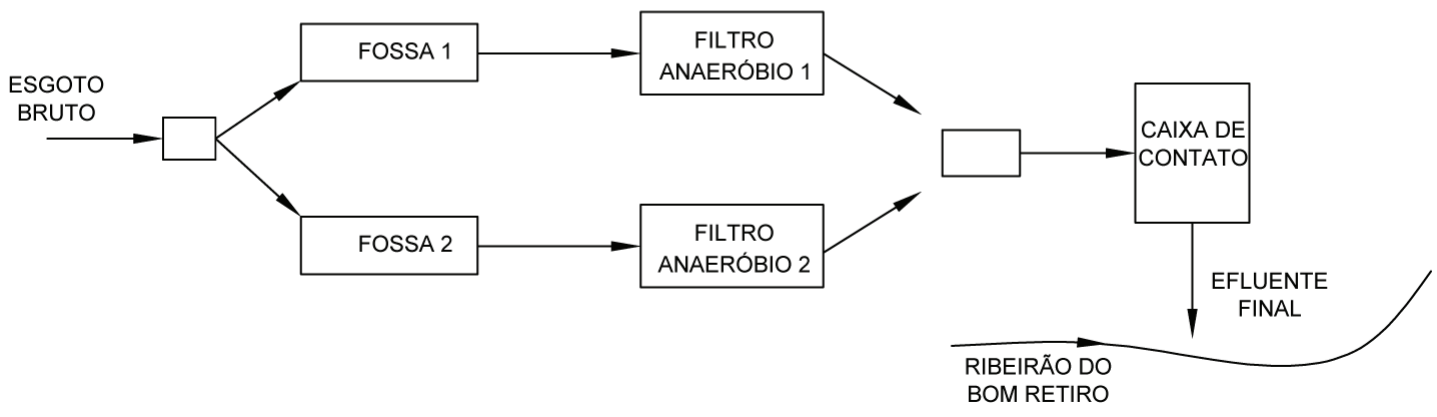

MODIFICAÇÃO REALIZADA NO ANO DE 2010

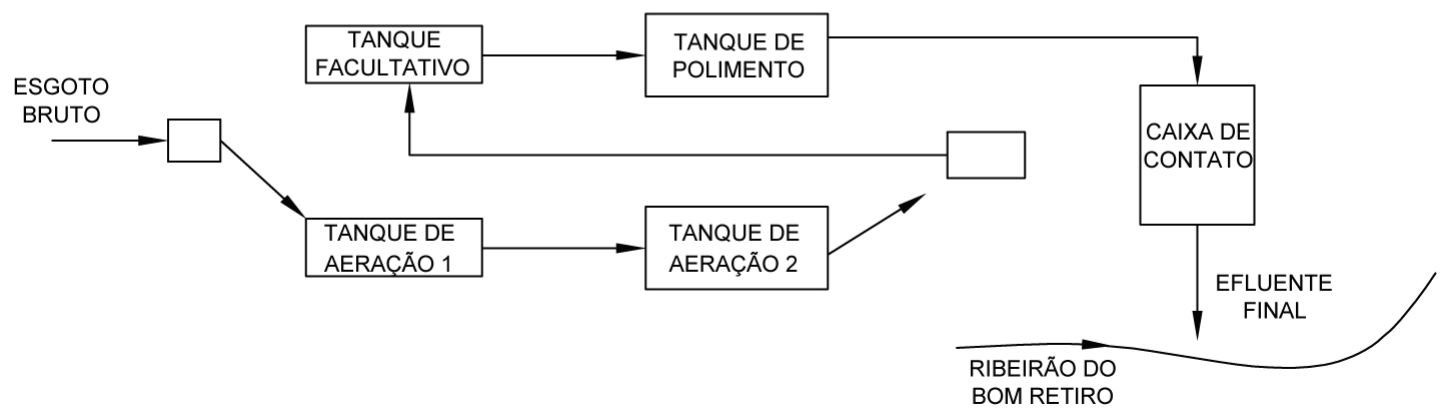

Figura 1 - Croqui do sistema de tratamento de esgoto antes e após a modificação.

\subsection{Planejamento do estudo na ETE do Dis-} trito Bom Retiro

Foi avaliado nas três etapas o sistema de aeração nos tanques de aeração (TA I e TA II). A submergência e a inclinação dos aeradores para todas as etapas foram de $0,90 \mathrm{~m}$ e $0^{\circ}$ com a horizontal, respectivamente. A Tabela 2 mostra o $n^{\circ}$ de aeradores e a potência aplicada em cada etapa do estudo. As Figuras 2, 3,4 e 5 mostram as unidades já modificadas e em operação.

\section{Resultados e discussão}

Baseado nos resultados obtidos de uma série histórica referente ao período de 2002 a 2008 efetuou-se o cálculo da eficiência do Tanque Séptico seguido de Filtro Anaeróbio da ETE Bom Retiro em relação à remoção da DBO. Os resultados são pontuais e estão na Tabela 3. De acordo com os dados encontrados, a eficiência média do sistema era de $61,18 \%$ de remoção de DBO, valores esses dentro do esperado de acordo com Hartmann et al. (2009) 


\begin{tabular}{|c|c|c|c|}
\hline & T.A I & T.A II & Período \\
\hline Volume $\left(\mathrm{m}^{3}\right)$ & 98,58 & 164,67 & \\
\hline \multicolumn{4}{|l|}{ Etapa I } \\
\hline Aerador $5 \mathrm{CV}$ & 2 unidades & 2 unidades & \multirow{3}{*}{ Jan. à Jul. 2011} \\
\hline Potência (CV) & 10 & 10 & \\
\hline D.P $\left(\mathrm{W} / \mathrm{m}^{3}\right)$ & 74 & 45 & \\
\hline \multicolumn{4}{|l|}{ Etapa II } \\
\hline Aerador (3 CV) & 3 unidades & - & \multirow{4}{*}{ Ago. 2011 à Jan. 2012} \\
\hline Aerador (2 CV) & - & 3 unidade & \\
\hline Potência (CV) & 9 & 6 & \\
\hline D.P $\left(\mathrm{W} / \mathrm{m}^{3}\right)$ & 67 & 27 & \\
\hline \multicolumn{4}{|l|}{ Etapa III } \\
\hline Aerador (5 CV) & 1 unidade & 1 unidade & \multirow{3}{*}{ Fev. à Ago. 2012} \\
\hline Potência (CV) & 5 & 5 & \\
\hline D.P $\left(\mathrm{W} / \mathrm{m}^{3}\right)$ & 37 & 22 & \\
\hline
\end{tabular}

Tabela 2 - Etapas do experimento

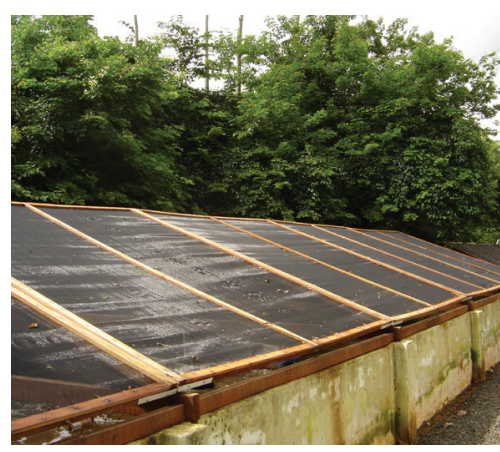

Figura 2 - Tanques de aeração I e II

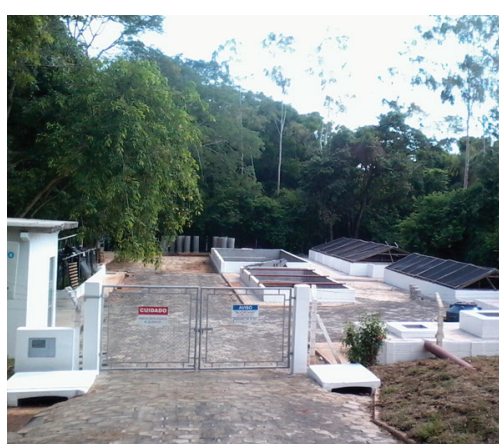

Figura 4 - Modificação concluída

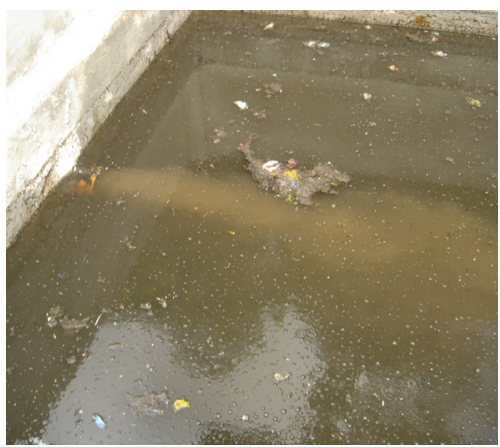

Figura 3 - Tanque Facultativo

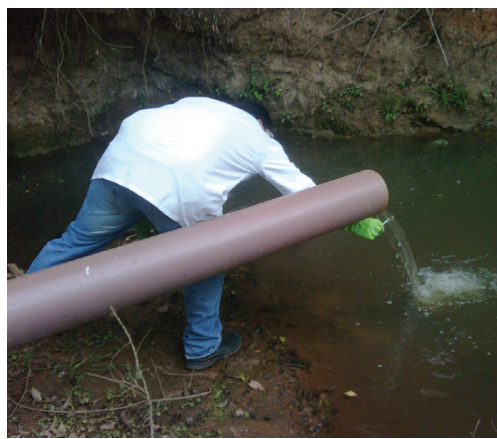

Figura 5 - Monitoramento do efluente 


\begin{tabular}{c|c|c} 
Data & DBO Afluente $\left(\mathrm{mg} \mathrm{O}_{2} \mathrm{~L}^{-1}\right)$ & DBO Efluente $\left(\mathrm{mg} \mathrm{O}_{2} \mathrm{~L}^{-1}\right)$ \\
\hline 04.12 .2002 & 730 & 247 \\
\hline 16.09 .2003 & 708 & 323 \\
\hline 20.09 .2005 & 744 & 250 \\
\hline 08.03 .2006 & 794 & 345 \\
\hline 27.03 .2007 & 742 & 395 \\
\hline 31.07 .2007 & 892 & 260 \\
\hline 27.02 .2008 & 603 & 220 \\
\hline 22.10 .2008 & 683 & 240 \\
\hline
\end{tabular}

Fonte: Divisão de Controle Sanitário - Sabesp, 2010

Tabela 3 - Valores da DBO afluente e efluente da ETE Bom Retiro, Angatuba, SP

5.1 Valores da DBO afluente da ETE Bom Retiro - Angatuba-SP

Para cálculo da carga orgânica afluente e para o dimensionamento do sistema de aeração, considerou-se como DBO afluente a média aparada obtida na Tabela 04, período de Agosto de 2010 a Fevereiro de 2012 com coletas pontuais na frequência semanal. Nesse cálculo foram desprezados $12 \%$ dos maiores e $12 \%$ dos menores valores. A média aparada a $24 \%$ da DBO afluente e o desvio padrão foi de $674 \pm 137 \mathrm{mg} / \mathrm{L}^{-1}$. (valores desprezados em vermelho)

\section{2 - Contribuição da vazão de esgoto estimada} no Distrito Bom Retiro.

Preliminarmente em 2010 para elaboração da concepção da proposta de modificação, avaliou- -se a estimativa de contribuição de esgoto, por meio de uma série histórica de dados referentes ao Volume Consumido Micromedido (VCM), o número de ligações, coeficiente de retorno e o número de habitantes por domicílio conforme dados do (IBGE, 2010). Os resultados estão na Tabela 05.

Posteriormente quando foi iniciada a operação do sistema modificado, foram registrados dados de vazões pontuais medidas em dias e horários diferentes, durante os meses de Janeiro a Julho de 2011 conforme Tabela 6.

Para referendar, o parâmetro de dimensionamento adotado (vazão da contribuição de esgoto ), verificou-se a mesma série histórica para 2011 conforme Tabela 7 e os resultados apresentados estão compatíveis com a vazão adotada.

\begin{tabular}{c|c|c|c|c|c} 
Data & DBO & Data & DBO & Data & DBO \\
\hline 06.08 .2010 & 763 & 11.05 .2011 & 663 & 28.12 .2011 & 704 \\
\hline 10.08 .2010 & 763 & 24.05 .2011 & 582 & 03.01 .2012 & 905 \\
\hline 17.08 .2010 & 460 & 31.05 .2011 & 744 & 10.01 .2012 & 844 \\
\hline 24.08 .2010 & 703 & 20.06 .2011 & 643 & 11.01 .2012 & 956 \\
\hline 01.09 .2010 & 683 & 28.06 .2011 & 884 & 12.01 .2012 & 563 \\
\hline 15.09 .2010 & 984 & 26.07 .2011 & 663 & 16.01 .2012 & 764 \\
\hline 29.09 .2010 & 804 & 09.08 .2011 & 905 & 26.01 .2012 & 400 \\
\hline 19.09 .2010 & 442 & 20.09 .2011 & 764 & 02.02 .2012 & 503 \\
\hline 28.10 .2010 & 864 & 25.10 .2011 & 350 & 06.02 .2012 & 400 \\
\hline 14.04 .2011 & 462 & 20.12 .2011 & 764 & 07.02 .2012 & 460 \\
\hline 28.04 .2011 & 442 & 21.12 .2011 & 824 & 08.02 .2012 & 543
\end{tabular}

Tabela 4 - DBO afluente em mg O2. L $^{-1}$ da ETE Bom Retiro - Angatuba SP 


\begin{tabular}{|c|c|c|c|c|c|c|}
\hline Mês & VCM m ${ }^{3}$ mês $^{-1}$ & $\begin{array}{c}N^{\circ} \text { Ligacções de } \\
\text { água }\end{array}$ & $\begin{array}{c}N^{\circ} \text { Ligações de } \\
\text { esgotos }\end{array}$ & $\begin{array}{c}\text { Consumo per } \\
\text { capita L.hab }{ }^{-1} \mathrm{~d}^{-1}\end{array}$ & $\begin{array}{l}\text { Esgoto VCM } \\
0.8 \mathrm{~m}^{3} \mathrm{mês}^{-1}\end{array}$ & $Q_{\text {esgoto }}$ L.s. \\
\hline $\operatorname{Jan} / 10$ & 6.193 & 586 & 542 & 108,22 & 4582 & 1,71 \\
\hline Fev/10 & 5.781 & 590 & 542 & 111,09 & 4245 & 1,75 \\
\hline Mar/10 & 6.164 & 591 & 543 & 106,81 & 4530 & 1,69 \\
\hline $\mathrm{Abr} / 10$ & 6.386 & 592 & 544 & 114,13 & 4694 & 1,81 \\
\hline Mai/10 & 5.721 & 591 & 546 & 99,13 & 4399 & 1,64 \\
\hline Jun/10 & 5.436 & 594 & 548 & 96,84 & 4115 & 1,60 \\
\hline Jul/10 & 6.496 & 595 & 552 & 111,80 & 5016 & 1,93 \\
\hline Ago/10 & 5.015 & 595 & 554 & 86,31 & 3615 & 1,39 \\
\hline Set $/ 10$ & 6.242 & 595 & 554 & 111,01 & 4804 & 1,79 \\
\hline Out/10 & 5.958 & 595 & 555 & 102,54 & 4438 & 1,66 \\
\hline Nov/10 & 6.166 & 596 & 556 & 109,48 & 4594 & 1,77 \\
\hline Dez/10 & 6.992 & 597 & 556 & 119,94 & 5209 & 1,94 \\
\hline \multicolumn{6}{|l|}{ Média } & $1,72 \pm 0,13$ \\
\hline
\end{tabular}

Tabela 5 - Consumo de água, ligações de esgoto e estimativa de vazão de esgoto

\begin{tabular}{|c|c|c|}
\hline Data 2011 & Horário & $\begin{array}{c}\text { Vazão afluente } \\
1 . \mathrm{s}^{-1}\end{array}$ \\
\hline 11.01 .2011 & 16h 00min & 1,91 \\
\hline 20.01 .2011 & 10h 00min & 1,63 \\
\hline 25.01 .2011 & $11 \mathrm{~h} 00 \mathrm{~min}$ & 1,72 \\
\hline 01.02 .2011 & $11 \mathrm{~h} 00 \mathrm{~min}$ & 1,71 \\
\hline 16.02 .2011 & $11 \mathrm{~h} 00 \mathrm{~min}$ & 1,50 \\
\hline 22.02 .2011 & $11 \mathrm{~h} 00 \mathrm{~min}$ & 1,83 \\
\hline 02.03 .2011 & $11 \mathrm{~h} 45 \mathrm{~min}$ & 2,00 \\
\hline 11.03 .2011 & $11 \mathrm{~h} 00 \mathrm{~min}$ & 1,91 \\
\hline 14.03 .2011 & $16 \mathrm{~h} 00 \mathrm{~min}$ & 2,00 \\
\hline 21.03 .2011 & $11 \mathrm{~h} 00 \mathrm{~min}$ & 1,64 \\
\hline 25.03 .2011 & 15h 30min & 1,45 \\
\hline 01.04 .2011 & $16 \mathrm{~h} \mathrm{00min}$ & 1,70 \\
\hline 14.04 .2011 & 10h $00 \mathrm{~min}$ & 1,43 \\
\hline 28.04 .2011 & 16h 30min & 1,54 \\
\hline 11.05 .2011 & 16h 00min & 1,72 \\
\hline 24.05 .2011 & $11 \mathrm{~h} 00 \mathrm{~min}$ & 1,32 \\
\hline 07.06 .2011 & 10h 35min & 1,25 \\
\hline 20.06 .2011 & $16 \mathrm{~h} 45 \mathrm{~min}$ & 1,60 \\
\hline 21.06.2011 & $17 \mathrm{~h} 00 \mathrm{~min}$ & 1,69 \\
\hline 28.06.2011 & $12 \mathrm{~h} 00 \mathrm{~min}$ & 1,74 \\
\hline 07.07 .2011 & $16 \mathrm{~h} 00 \mathrm{~min}$ & 1,27 \\
\hline 15.07.2011 & $16 \mathrm{~h} 00 \mathrm{~min}$ & 1,80 \\
\hline 19.07.2011 & $16 \mathrm{~h} 30 \mathrm{~min}$ & 2,20 \\
\hline 22.07.2011 & $16 \mathrm{~h} 00 \mathrm{~min}$ & 1,70 \\
\hline 24.07.2011 & 10h $00 \mathrm{~min}$ & 1,67 \\
\hline 26.07.2011 & $17 \mathrm{~h} 00 \mathrm{~min}$ & 2,00 \\
\hline
\end{tabular}

Tabela 6 - Vazão de esgoto medida 


\begin{tabular}{c|c|c|c|c|c|c} 
Mês & VCM m ${ }^{3} \mathrm{mês}^{-1}$ & $\begin{array}{c}\mathrm{N}^{\circ} \text { Ligações de } \\
\text { água }\end{array}$ & $\begin{array}{c}\mathrm{N}^{\circ} \text { Ligações de } \\
\text { esgotos }\end{array}$ & $\begin{array}{c}\text { Consumo per } \\
\text { capita L.hab } \mathrm{d}^{-1}\end{array}$ & $\begin{array}{c}\text { Esgoto VCM } \\
0.8 \mathrm{~m}^{3} \mathrm{mês}^{-1}\end{array}$ & $\begin{array}{c}\text { Vazão de } \\
\text { esgoto L. } \mathrm{s}^{-1}\end{array}$ \\
\hline Jan/11 & 6.118 & 597 & 555 & 104,94 & 4.550 & 1,70 \\
\hline Fev/11 & 6.281 & 600 & 556 & 118,69 & 4.656 & 1,92 \\
\hline Mar/11 & 6.581 & 599 & 555 & 112,50 & 4.878 & 1,82 \\
\hline Abr/11 & 6.154 & 602 & 555 & 108,19 & 4.539 & 1,75 \\
\hline Mai/11 & 6.114 & 607 & 558 & 103,15 & 4.496 & 1,68 \\
\hline Jun/11 & 6.240 & 606 & 558 & 108,96 & 4.596 & 1,77 \\
\hline Jul/11 & 5.970 & 607 & 562 & 100,72 & 4.422 & 1,65 \\
\hline Ago/11 & 5.848 & 609 & 561 & 98,35 & 4.310 & 1,61 \\
\hline Set/11 & 6.953 & 613 & 563 & 120,02 & 5.109 & 1,97 \\
\hline Out/11 & 6.744 & 615 & 564 & 112,31 & 4.948 & 1,85 \\
\hline Nov/11 & 6.345 & 619 & 568 & 108,46 & 4.658 & 1,80 \\
\hline Dez/11 & 6.359 & 620 & 569 & 105,03 & 4.669 & 1,74 \\
\hline Média & & & & & & $1,77 \pm 0,11$ \\
\hline
\end{tabular}

Fonte: Sabesp. 2011

Tabela 7 - Consumo de água, ligações e estimativa de vazão de esgoto

\subsection{Dados do sistema Bom Retiro - Base de cálculo para a modificação}

O projeto de modificação do sistema de tratamento foi baseado nos parâmetros a seguir:

Número de ligação de esgoto

Atendimento de esgoto - 2011

Extensão da rede - 2011

No Habitantes/residência

Previsão de crescimento

Atendimento de esgoto -2030

Contribuição de esgoto medido

Contribuição de esgoto estimada

Contribuição de esgoto estimada

Vazão média adotada

Vazão média estimada

DBO afluente (média)

Sólidos Totais ( média)

Sólidos em suspensão Total

Carga orgânica afluente calculada

Carga orgânica afluente calculada
$558(06 / 2011)$

1758 habitantes

$5080 \mathrm{~m}$

3,15 (IBGE - Censo demográfico 2010)

1,42\% a.a (IBGE - Censo demográfico 2010)

2.256 habitantes - alcance do projeto 20 anos

$1,70 \pm 0,24 \mathrm{Ls}^{-1}$

5.970

$1,72 \pm 0,13 \mathrm{Ls}^{-1}(2010)$

$1,75 \mathrm{~L} \mathrm{~s}-1=151,20 \mathrm{~m}^{3} \mathrm{~d}^{-1}$ (início de plano)

$2,80 \mathrm{~L} \mathrm{~s}-1=242,40 \mathrm{~m}^{3} \mathrm{~d}^{-1}$ (final de plano)

$674 \pm 137 \mathrm{mg} / \mathrm{L}^{-1}$

$1061 \pm 285 \mathrm{mg} / \mathrm{L}^{-1}$

$406 \pm 60 \mathrm{mg} / \mathrm{L}^{-1}$

$163,38 \mathrm{Kg} \mathrm{DBO} \mathrm{d}^{-1}$ (final plano)

101,91 kg DBO d ${ }^{-1}$ (início de plano) 


\subsection{Dimensionamento - Cálculos Básicos}

\subsubsection{Potência de aeração}

Com a modificação do sistema objetivou-se a remoção da carga orgânica de 10\% no tratamento preliminar e no tratamento proposto remoção maior ou igual a 85\%. A carga orgânica considerada após tratamento preliminar foi de $91,72 \mathrm{Kg} \mathrm{DBO} \mathrm{d}^{-1}$. Para remoção da carga no tratamento de 77,96 Kg DBO d ${ }^{-1}$, a necessidade de oxigênio e a potência aplicada foram calculadas a seguir. Adotou-se como requisitos médios de oxigênio com nitrificação para lodo ativado com aeração prolongada, 1,5 a $1,8 \mathrm{Kg} \mathrm{O}_{2} \mathrm{Kg}^{-1} \mathrm{DBO}$ (Fonte: Arceivala (1981), Orhon \& Artan (1994), apud Von Sperling,1997). Adotou-se 1,5 $\mathrm{Kg} \mathrm{O}_{2}$ $\mathrm{Kg}^{-1} \mathrm{DBO}$.

Necessidade de oxigênio $=116,94 \mathrm{Kg} \mathrm{O}_{2} \mathrm{~d}^{-1}=$ $4,87 \mathrm{Kg} \mathrm{O}_{2} \mathrm{~h}^{-1}$

Taxa de transferência de oxigênio do aerador (submerso) para condição padrão: $\mathrm{N}_{0}=1,2 \mathrm{Kg} \mathrm{O}_{2}$ $\mathrm{kWh}^{-1}$;

Para as condições de campo têm-se: $\mathrm{N}=\mathrm{N}_{\mathrm{o}} \mathrm{x}$ $\lambda$. A equação 1é utilizada para cálculo do fator de correção da condição padrão para condição de campo $(\lambda)$.

$$
\frac{\lambda=\alpha(\beta . \mathrm{CSW}-\mathrm{CL}) 1,02^{\mathrm{T}-20}}{9,17}
$$

(METCALF \& EDDY, 1991)

Equação (1) onde:

$\alpha$ : relação entre a taxa de transferência de $O_{2}$ para esgoto pela taxa de transferência para água limpa $(0,85)$; ( Metcalf \& Eddy apud Von Sperling, 2002)

$C_{L}$ : concentração de $O D$ no tanque de aeração $\left(1,5 \mathrm{mg} / \mathrm{L}^{-1}\right)$

$C_{S W}$ : concentração de saturação de $O_{2}$ para altitude e temperatura do local (620 m e $\left.27^{\circ} \mathrm{C}-7,6 \mathrm{mg} / \mathrm{L}^{-1}\right)$ (VON SPERLING, 2002)

$\beta$ : relação entre o $O D$ de saturação para esgoto pelo $O D$ de saturação para água limpa $(0,95)$. (Metcalf \& Eddy apud Von Sperling, 2002)

Dessa forma a potência de aeração necessária seria de $6,67 \mathrm{~kW}$ aproximadamente $10 \mathrm{CV}$. O estudo foi desenvolvido em três etapas distintas, nas quais foram alteradas as potências dos aeradores nos tanques de aeração.

\subsubsection{Instalações dos aeradores na $1^{\mathrm{a}}$ Etapa do estudo}

Na primeira etapa do estudo foram utilizados dois aeradores de $5 \mathrm{CV}$ em cada tanque de aeração, conforme Figura 06. Foi utilizada cobertura com sombrite para proteger o sistema de aeração, para evitar que folhas ou galhos da mata ao redor da estação adentrassem ao sistema e comprometessem o funcionamento dos aeradores. Nesta $1^{\text {a }}$ Etapa adotou-se um acréscimo de 100\% da potência necessária para remoção de 85\% da carga de DBO afluente conforme item 3.2.

\subsubsection{Instalações dos aeradores na $2^{\mathrm{a}}$ Etapa do estudo}

Na segunda etapa do estudo foram utilizados três aeradores de 3 CV no TA I e três aeradores de 2 CV no TA II, conforme Figura 07. Nesta etapa adotou-se acréscimo de $50 \%$ da potência necessária para remover $85 \%$ da carga de DBO afluente, conforme item 3.2 .
AERADOR SUBMERSO DE 5 CV CADA UNIDADE

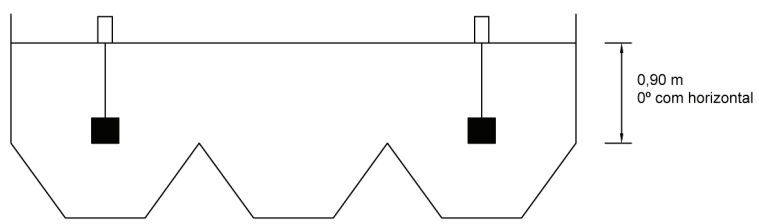

AERADOR SUBMERSO DE 5 CV CADA UNIDADE

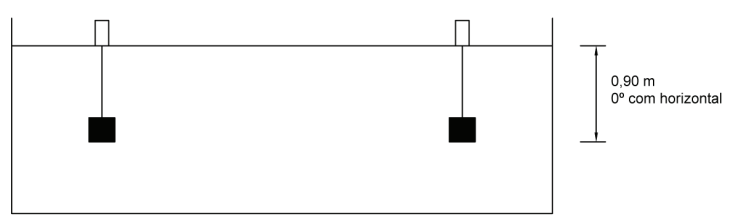

Figura 6 - Detalhe dos tanques de aeração I e II corte longitudinal. 
AERADOR SUBMERSO DE 3 CV CADA UNIDADE

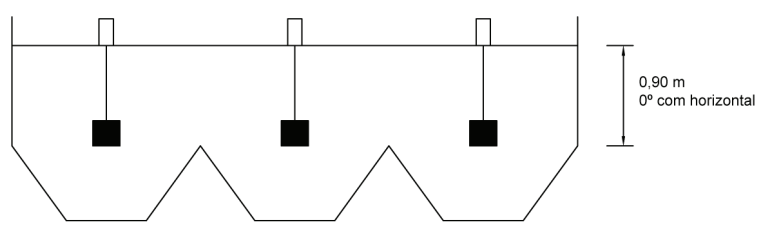

AERADOR SUBMERSO DE 2 CV CADA UNIDADE

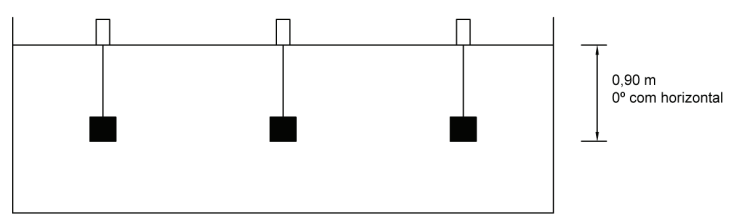

Figura 7 - Detalhe dos tanques de aeração I e II

AERADOR SUBMERSO DE $5 \mathrm{CV}$

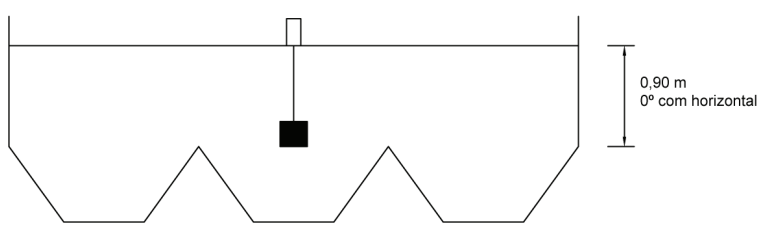

AERADOR SUBMERSO DE 5 CV

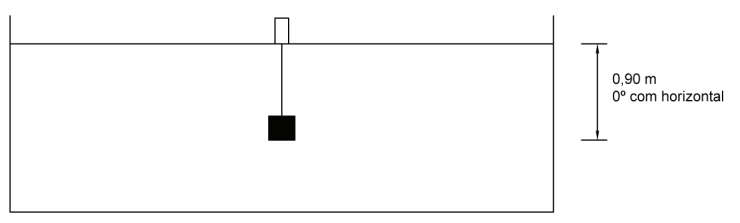

Figura 8 - Detalhe dos tanques de aeração I e II - $3^{\text {a }}$ Etapa do experimento

\subsubsection{Instalações dos aeradores na $3^{\mathrm{a}}$ Etapa do estudo}

$\mathrm{Na}$ terceira etapa do estudo, foi utilizado um aerador de 5 CV em cada tanque de aeração, conforme Figura 08. Nesta etapa adotou-se a densidade de potência necessária calculada para remover $85 \%$ da carga de DBO afluente determinada, conforme item 3.2.

\section{5 Avaliação da DBO em todas as unidades do processo}

Na Figura 09 estão os valores da DBO e desvios padrões nas várias unidades do processo: saída do TA-I, saída do (TA-II), saída do Tanque Facultativo (T.F) e saída do Tanque de Polimento (T.P), referente a $1^{\text {a }}$ Etapa do estudo.

\subsubsection{Remoção da DBO no Tanque de Aeração I e II}

Na $1^{\text {a }}$ Etapa do estudo, foi constatado que a carga orgânica remanescente, após passagem pelos tanques de aeração I e II, permitiu bom desempenho dos tanques facultativos e de po- limento, com relação a redução da DBO total ( volátil e fixa), em virtude da carga volumétrica aplicada ( Kg DBO. $\mathrm{m}^{-3}$.dia ) nos tanques apresentar valores adequados.

\subsubsection{Taxa de aplicação volumétrica (Lv) nos Tan-} ques Facultativo e de Polimento

Com as características do sistema modificado próximas a um lodo ativado com aeração prolongada $(\mathrm{TDH}=28 \mathrm{~h})$ a $\mathrm{Lv}$ recomendada é de 0,16 a 0,40 Kg DBO. $\mathrm{m}^{-3}$.dia (CETESB, 1988).

A remoção da DBO nos tanques de aeração foi: $\mathrm{TA} \mathrm{I}=67,1 \%$ e TA II $=34,7 \%$

\% Remoção em TAI + TAII = 674 - (674) [(10,671) $(1-0,347)] / 674=79,2 \%$

Remoção de $10 \%$ da carga de DBO no tratamento preliminar:

C.O = Início de plano: $101,91 \mathrm{~kg}$ DBO dia $^{-1} \mathrm{X}$ $0,90=91,72 \mathrm{~kg}$ DBO dia ${ }^{-1}$

C.O (Kg DBO $\mathrm{d}^{-1}$ ) remanescente para os Tanques Facultativo e de Polimento $=91,72 \mathrm{Kg}$ DBO $\mathrm{d}^{-1}$ X 0, $208=19,08 \mathrm{Kg}^{\mathrm{DBO}} \mathrm{d}^{-1}$ 
MÉDIA +/- 1 DESVIO PADRÃO

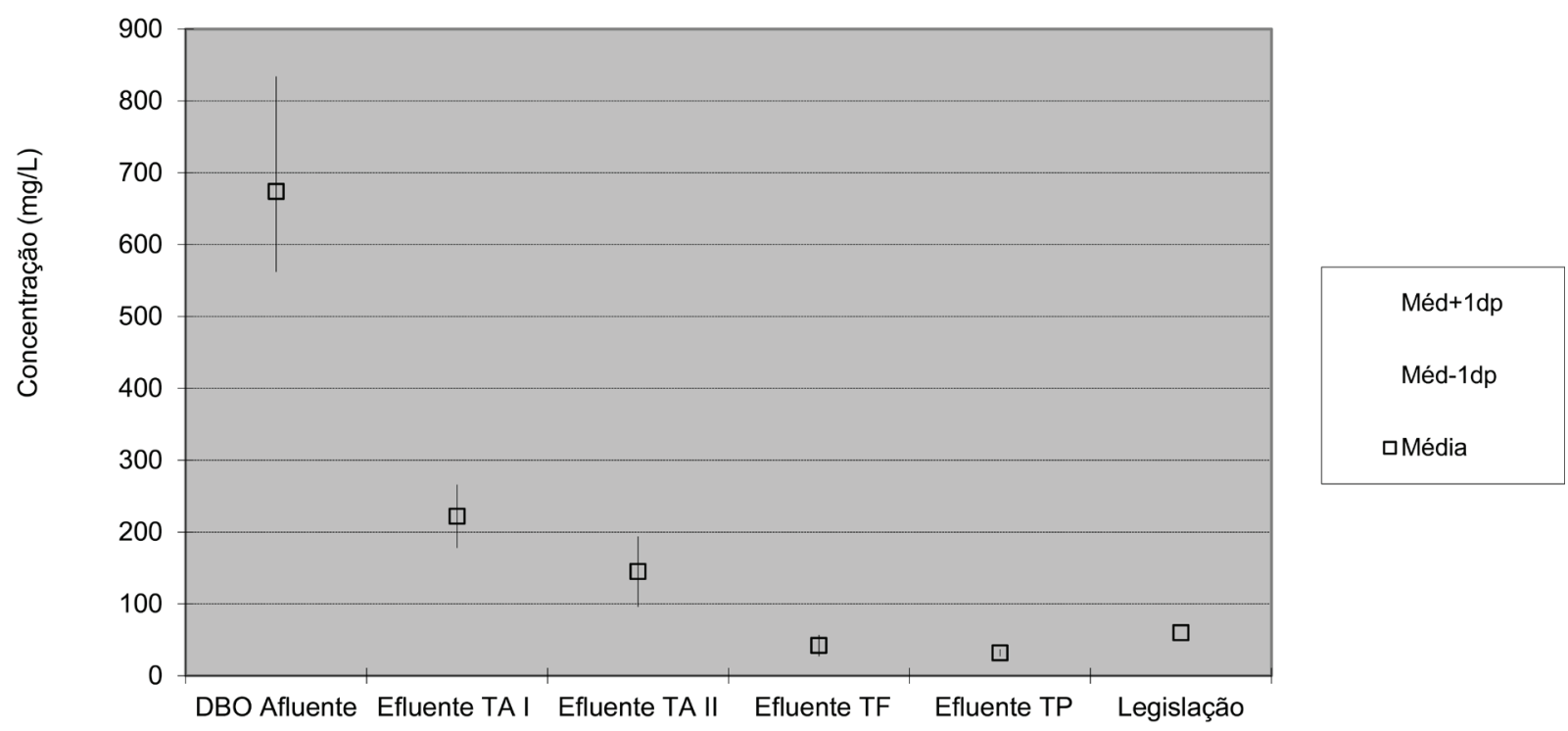

Figura 9 - Valores de DBO nas etapas do processo ( TA I, TA II, TF e TP)

Volume de sedimentação $=$ Tanque Facultativo + Tanque de polimento $=175,20 \mathrm{~m}^{3}$

$$
\begin{array}{r}
L v=\frac{\text { C. O }}{V}=\frac{19,70 \mathrm{~kg} \mathrm{DBOd}^{-1}}{175,20 \mathrm{~m}^{3}}=0,11 \mathrm{Kg} \mathrm{DBOm}^{-3} \mathrm{~d}^{-1} \\
\text { Equação (2) }
\end{array}
$$

Os resultados foram satisfatórios na $1^{\mathrm{a}}$ Etapa do estudo, com relação à remoção de carga orgânica no Tanque Facultativo e Tanque de Polimento, devido à boa remoção de carga orgânica nos tanques de aeração. A taxa volumétrica aplicada nos tanques facultativo e de polimento foi de $0,11 \mathrm{Kg}^{\mathrm{DBO}} \mathrm{m}^{-3} \mathrm{~d}^{-1}$, abaixo dos valores apre- sentados na literatura, tanto para processo de tratamento aeróbio como para processo anaeróbio (CETESB, 1988).

Média da \% de remoção para DBO no Tanque Facultativo $(\mathrm{TF})=71 \%$

Média da \% de remoção para DBO no Tanque de Polimento (TP) $=24 \%$

\% Remoção TF + TPI = $145-(145)(1-0,71))$ $1-0,24)] / 145=78 \%$

\section{6 Avaliação do SST em todas as unidades do processo}

Na Figura 10 estão os valores de SST nas várias etapas do processo. A remoção média de SST na $1^{\text {a }}$ Etapa do experimento foi de 89,6\%. Onde TA I e TA 2 - Saídas dos tanques de aeração I e II, respectivamente e TF e TP Saída dos tanques facultativo e de polimento. 


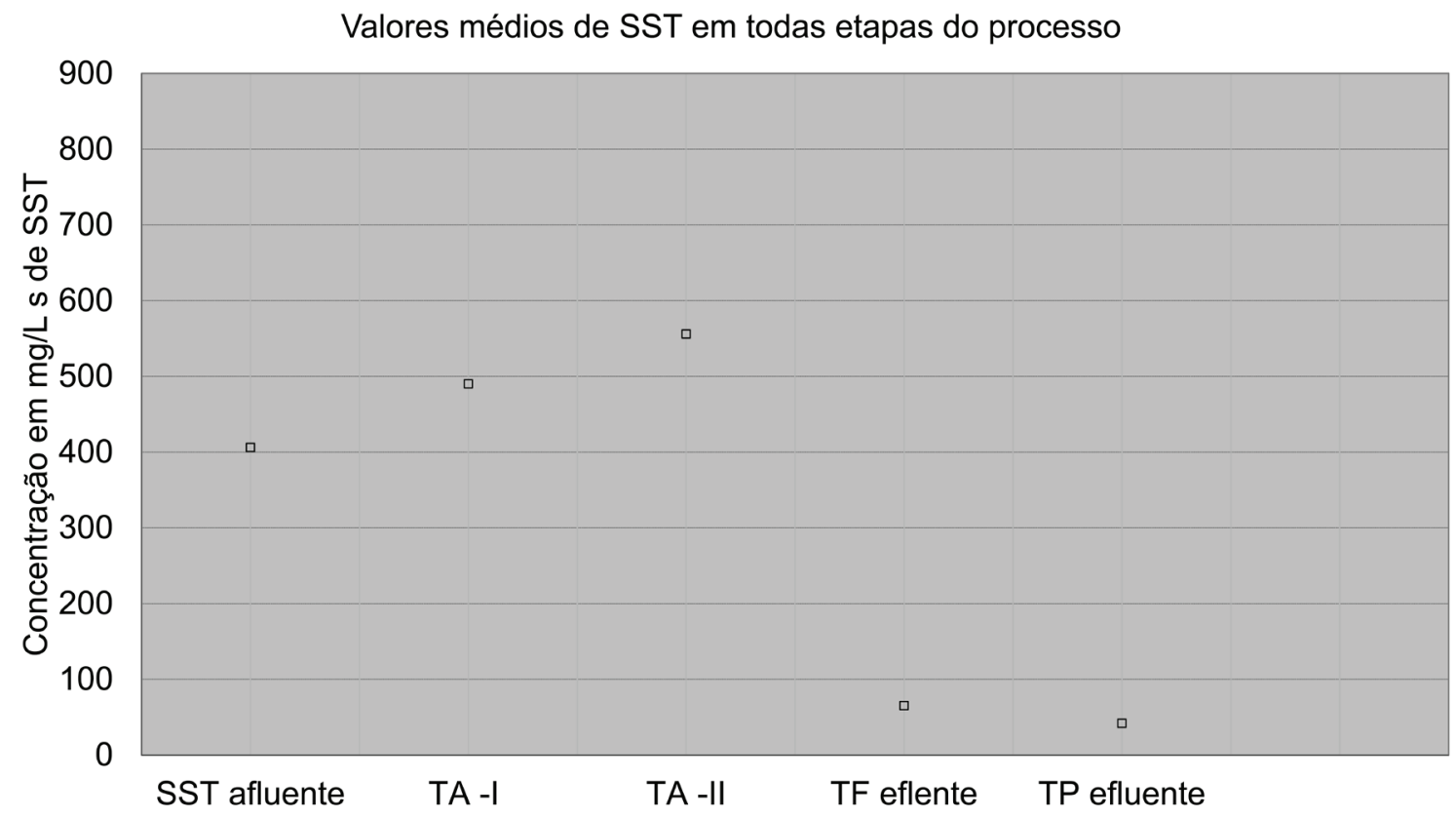

Figura 10 - Valores do Teor de SST nas etapas do processo (TAI, TAII, TF e TP)

\section{Variação do Teor de OD em função do tempo}

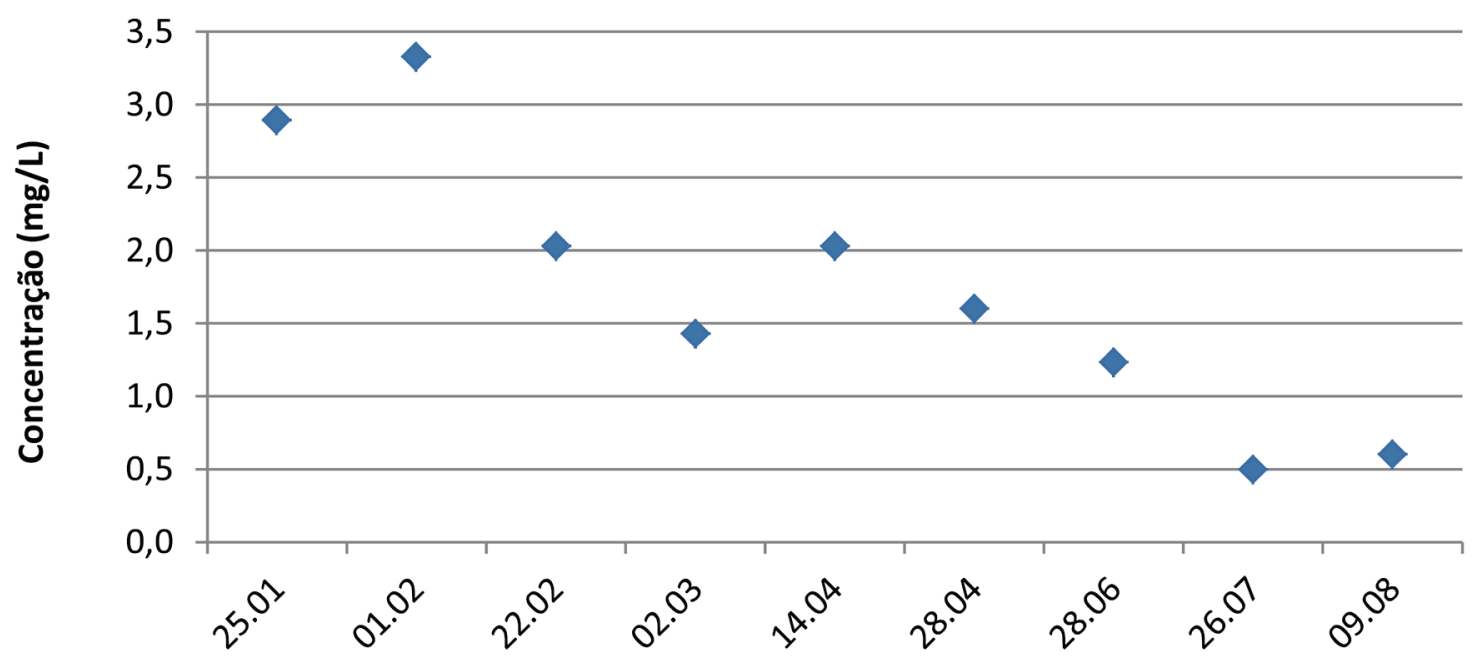

Fonte: Divisão de Controle Sanitário - Sabesp, 2011 
5.7 Residual de oxigênio dissolvido na saída do tanque de Polimento

O teor de OD na saída do Tanque de Polimento referente a $1^{\text {a }}$ etapa do estudo está mostrado na Figura 11. Durante essa etapa, a média obtida foi de $1,68 \mathrm{mg} / \mathrm{L}^{-1}$.

\subsection{Consumo de energia na $1^{\mathrm{a}}$ etapa do estudo} (Janeiro a Julho de 2011)

O consumo médio mensal de energia elétrica do sistema modificado foi de $10.698 \mathrm{kWh}$. Os meses avaliados estão no relatório de controle do consumo de energia da Sabesp de 2011.

O Consumo de $73 \mathrm{kWh} \mathrm{hab}^{-1} \mathrm{ano}^{-1}$ nesta etapa do estudo, está acima dos valores mencionados na literatura para lagoas aeradas que variam de 16 a $22 \mathrm{kWh} \mathrm{hab}^{-1} \mathrm{ano}^{-1}$. Com valores de 60 a $90 \mathrm{mg} / \mathrm{L}$ para SST efluente e valores de 50 a $80 \mathrm{mg} / \mathrm{L}^{-1}$ para DBO efluente (Von Sperling,2005). O sistema modificado apresentou valor médio de SST efluente de $42 \pm 20 \mathrm{mg} / \mathrm{L}^{-1}$ e DBO efluente de $32 \pm$
$5 \mathrm{mg} / \mathrm{L}^{-1}$, ambientalmente mais favoráveis.

\subsection{Custo para modificação da ETE Bom Retiro}

Os itens contemplados para a modificação da ETE estão no relatório de Gestão de Empreendimentos Sabesp, 2011. O custo total foi de R\$246.000,00 $\left(\mathrm{P}_{0}=\right.$ Junho/2011). Em torno de R\$ 140,00 por habitante, para um alcance de projeto de 20 anos. Além das opções abaixo apresentarem custo de implantação mais elevado, o sistema modificado mostrou-se mais eficiente quando comparados com valores de literatura, para os resultados do efluente com relação à remoção de DBO e SST. A Tabela 08 mostra custo de outras opções também possíveis para o espaço disponível.

\subsection{Segunda e terceira etapa do estudo na ETE Bom Retiro}

Todos os ensaios e testes realizados na $1^{\mathrm{a}}$ etapa do estudo foram repetidos para as $2^{\mathrm{a}}$ e $3^{\mathrm{a}}$ etapas e os resultados estão na Tabela 09.

\begin{tabular}{l|cc}
\hline \multicolumn{1}{c|}{ Unidades } & Custo R\$ hab-1 & Custo da implantação (R\$) \\
\hline Lagoa aerada e decantação ( aerador submerso) & 292,05 & $513.432,90$ \\
\hline Lodo ativado-aeração prolongada- TA. anóxico & 542,33 & $953.416,14$ \\
\hline
\end{tabular}

Fonte: Gestão de Empreendimento - Sabesp, 2012

Tabela 8 - Custo de outras opções para adequação da ETE Bom Retiro

\begin{tabular}{|c|c|c|c|}
\hline & $1^{\text {a }}$ Etapa & $2^{\mathrm{a}}$ Etapa & $3^{\text {a Etapa }}$ \\
\hline Potência aplicada & $20 \mathrm{CV}$ & $15 \mathrm{CV}$ & $10 \mathrm{CV}$ \\
\hline D.P $\left(W \cdot m^{-3}\right)$ & $59 \mathrm{~W} \cdot \mathrm{m}^{-3}$ & $47 \mathrm{~W} \cdot \mathrm{m}^{-3}$ & $29,5 \mathrm{~W} \cdot \mathrm{m}^{-3}$ \\
\hline Consumo de energia & $73 \mathrm{~kW} \cdot \mathrm{hab}^{-1} \cdot \mathrm{ano}^{-1}$ & $56 \mathrm{~kW} \cdot \mathrm{hab}^{-1} \cdot \mathrm{ano}^{-1}$ & 37 kW.hab-.ano-1 \\
\hline $\mathrm{DBO}_{5,20}$ efluente & $32 \pm 5 \mathrm{mgO} 2 . \mathrm{L}^{-1}$ & $22 \pm 5 \mathrm{mgO} 2 . \mathrm{L}^{-1}$ & 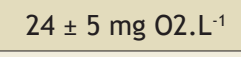 \\
\hline Remoção DBO nos TA & $79,2 \%$ & $86,7 \%$ & $86,2 \%$ \\
\hline $\begin{array}{l}\text { Remoção DBO nos T. Faculta- } \\
\text { tivo e polimento }\end{array}$ & $78,0 \%$ & $75,5 \%$ & $74,1 \%$ \\
\hline $\begin{array}{l}\text { Carga volumétrica nos Tan- } \\
\text { ques TF e TP (Lv) }\end{array}$ & $0,11 \mathrm{Kg} \cdot \mathrm{DBO}, \mathrm{m}^{3} \mathrm{~d}^{-1}$ & $0,07 \mathrm{Kg} \cdot \mathrm{DBO}, \mathrm{m}^{3} \cdot \mathrm{d}^{-1}$ & $0,07 \mathrm{Kg} \cdot \mathrm{DBO}, \mathrm{m}^{3} \mathrm{~d}^{-1}$ \\
\hline Remoção da DBO & $95,2 \%$ & $96,7 \%$ & $96,4 \%$ \\
\hline O.D saída tratamento & $2,0 \pm 0,7 \mathrm{mg} \cdot \mathrm{L}^{-1}$ & $3,6 \pm 1,2 \mathrm{mg} \cdot \mathrm{L}^{-1}$ & $1,91 \pm 1,1 \mathrm{mg} \cdot \mathrm{L}^{-1}$ \\
\hline Remoção de SST & $89,3 \%$ & $89,4 \%$ & $86,7 \%$ \\
\hline SST efluente & $42 \mathrm{mg} \cdot \mathrm{L}^{-1}$ & $39 \mathrm{mg} \cdot \mathrm{L}^{-1}$ & $54 \mathrm{mg} \cdot \mathrm{L}^{-1}$ \\
\hline
\end{tabular}

Fonte: Divisão de Controle Sanitário - Sabesp, 2012

Tabela 9 - Resumo dos resultados obtidos em todas as Etapas do experimento 


\subsection{Quantificação do lodo gerado nos Tanques} Facultativo e de Polimento

A Tabela 10 apresenta teor de sólidos médio do lodo nos tanques.

\begin{tabular}{|c|c|} 
Data da coleta & $\begin{array}{c}\text { Resultados de sólidos } \\
\text { totais (\%) }\end{array}$ \\
\hline 28.04 .2011 & 6,66 \\
\hline 23.08 .2011 & 5,41 \\
\hline 24.08 .2011 & 5,19 \\
\hline 09.09 .2011 & 6,92 \\
\hline 23.09 .2011 & 8,10 \\
\hline 07.11 .2011 & 4,40 \\
\hline
\end{tabular}

Fonte: Divisão de Controle Sanitário - Sabesp, 2011

Tabela 10 - Teor de sólidos totais médio no lodo durante a remoção

5.11.1 Remoção do lodo gerado nos tanques facultativo e de polimento

O lodo foi removido por meio de bombas submersíveis, condicionado com polímero orgânico sintético catiônico, em emulsão. Os contentores geotêxteis foram instalados em suportes metálicos com o fundo apoiado em

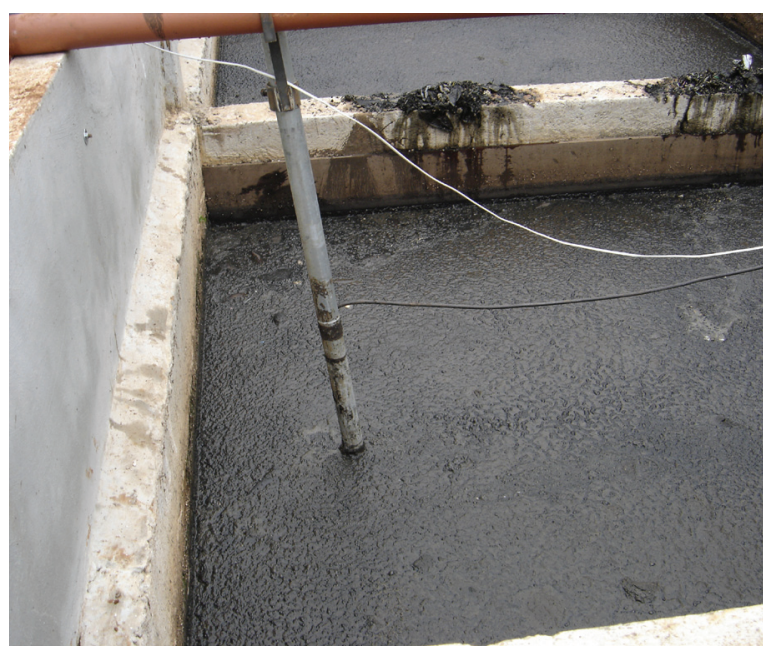

Figura 12 - Remoção do lodo dos tanques uma camada de brita 1 com 0,20 m de espessura em nível e fundo com declividade de $1 \%$ para coleta e retorno do drenado ao tanque de polimento. As Figuras 12 e 13 mostram o procedimento para remoção e acondicionamento do lodo.

Devido a pequena quantidade de lodo gerado anualmente, optou-se por eliminar o tratamento da fase sólida "in loco" e tratá-lo conjuntamente em ETE maior com sistema de desaguamento de lodo já implantado. Devido a proximidade o transporte pode ser feito por meio de caminhão limpa fossa. A alternativa se mostrou viável operacional e financeiramente. Neste caso o custo da modificação deverá ficar em torno de $\mathrm{R} \$ 106,00$ por habitante.

\section{Conclusões}

A modificação do sistema de tratamento de esgoto se mostrou viável para este caso, uma vez que todas as etapas do estudo apresentaram boa eficiência com relação à remoção de DBO e SST, além da eliminação da produção de gases mal cheirosos.

Os resultados obtidos nas diferentes etapas do estudo, para os parâmetros analisados mostraram que o sistema apresentou boa estabilidade para as condições operacionais adotadas.

A densidade de potência para a terceira etapa ficou em torno de 30 W. $\mathrm{m}^{-3}$ e o consumo de energia em $37 \mathrm{~kW} \cdot \mathrm{hab}^{-1} \cdot \mathrm{ano}^{-1}$, com possi-

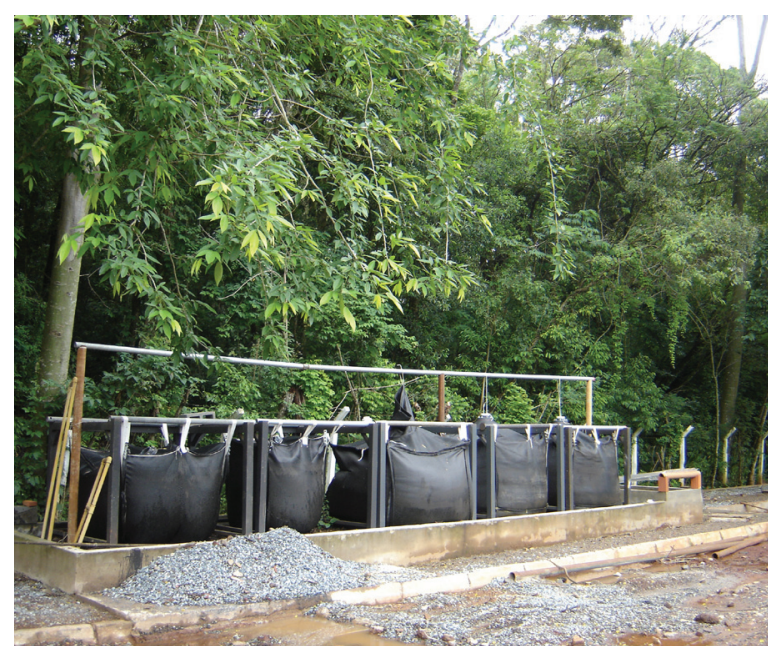

Figura 13 - Bags em desaguamento 
bilidade de redução desses parâmetros após avaliação por meio de testes de opções mais eficientes de aeração.

Houve atendimento aos aspectos legais, principalmente junto ao órgão ambiental e outras instâncias para obtenção de licença de operação.

Sugere-se a modificação como alternativa para sistemas semelhantes, ou seja, tanque séptico seguido de filtro anaeróbio, desde que sejam avaliados o tempo hidráulico no processo de aeração e a taxa de aplicação volumétrica no processo de sedimentação.

\section{Referências bibliográficas} ASSOCIAÇÃO BRASILEIRA DE NORMAS TÉCNICASNBR 12.209/2011:Elaboração de projetos hidráulico-sanitários de estações de tratamentode esgotos sanitários. Rio Janeiro, 1997.

BRASIL. Instituto Brasileiro de Geografia e Estatística (IBGE): Pesquisa Nacional de Saneamento, 2008.

BRASIL. Instituto Brasileiro de Geografia e Estatística (IBGE): Censo Demográfico, 2010,http://www.ibge.gov.br/ acesso 12.12 .2011

Centro de Pesquisas Metereológicas e Climáticas (CEPAGRI), acesso site http://www.cpa. unicamp.br/ em 10.01. 2012 as 17 h $00 \mathrm{~min}$

COMPANHIA DE SANEAMENTO DO ESTADO DE SÃO PAULO - SABESP, Relatório Gerencial da Unidade de Negócio do Alto Paranapanema, 2010.

COMPANHIA DE TECNOLOGIA E SANEAMENTO AMBIENTAL _ CETESB. Treinamento: Recuperação da qualidade das águas. Capítulo 7. São Paulo, 1988.

METCALF \& EDDY. Wastewater engineering: treatment, disposal and reuse. 3ed. 1991

JORDÃO, E. P. \& PESSÔA, C. A. Tratamento de Esgotos Domésticos. 5 ed. Rio de Janeiro, 2009. $940 \mathrm{p}$.

VON SPERLING, M. Lagoas de estabilização.

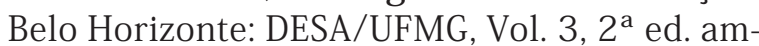
pliada, 2002.

VON SPERLING, M. Lodos ativados. Belo Horizonte: DESA/UFMG, Volume $4,2^{\mathrm{a}}$ ed. Am- pliada, 1997.

CLEVERSON, V, A; VON SPERLING, M; FERNANDO, F. Lodo de esgotos: Tratamento e disposição final. Belo Horizonte: Departamento de Engenharia Sanitária e ambiental-UFMG; Companhia de Saneamento do Paraná. 484p.

Wilks, D.S., Statistical methods in the atmospheric sciences an introduction. Academic Press, 467 p, 1995.

SOBRE OS AUTORES

Josué Tadeu Leite França*

Engenheiro Químico pela Fundação Armando Alvares Penteado (FAAP), M.Sc. e Doutor em Saneamento e Ambiente pela Faculdade de Engenharia Civil, Arquitetura e Urbanismo da UNICAMP e Engenheiro na Unidade do Alto Paranapanema, SABESP.

Ronaldo Stefanutti

Engenheiro Agrônomo, Professor Titular da Universidade Federal do Ceará.

Bruno Coraucci Filho

Engenheiro Civil, Professor Titular da Faculdade de Engenharia Civil, Arquitetura e Urbanismo - UNICAMP

Luccas Erickson Marinho

Bacharel em biotecnologia, Mestre em Engenharia Civil.

Gentil Tereziano Rodrigues

Técnico em Sistema de Saneamento na Unidade de Negócio do Alto Paranapanema, SABESP

Endereço para correspondência $\left({ }^{*}\right)$ :

Rua Marino Alves Palomo, 65 Jardim Dayse - CEP: 18.210-

290 - Itapetininga SP/Brasil- (15) 3272 - 6792 (15)

981220862 E-mail: jleitefra@ig.com.br 